\title{
RESEARCH
}

Open Access

\section{Reliability of MRI in detection and differentiation of acute neonatal/pediatric encephalopathy causes among neonatal/ pediatric intensive care unit patients}

Tamir A. Hassan ${ }^{*}$ and Nesreen Mohey

\begin{abstract}
Background: Causes of encephalopathy in neonates/pediatrics include hypoxic-ischemic injury (which is the most frequent cause and is defined as any impairment to the brain caused by insufficient blood flow and oxygenation), trauma, metabolic disorders, and congenital and infectious diseases. The aim of this study is to evaluate the value of MRI in detection and possible differentiation of different non-traumatic, non-infectious causes of acute neonatal/ pediatric encephalopathy among NICU/PICU patients.

Results: This retrospective study included 60 selected patients according to the study inclusion and exclusion criteria; all presented with positive MRI findings for non-traumatic, non-infectious acute brain injury. Females (32, $53.3 \%)$ were affected more than males $(28,46.7 \%)$ with a mean age of $1.1 \pm 1.02$ years; all presented with variable neurological symptoms and signs that necessitate neonatal intensive care unit/pediatric intensive care unit (NICU/ $\mathrm{PICU}$ ) admission. The final diagnosis of the study group patients were hypoxic ischemia injury (HII) in 39 patients (65\%), metachromatic leukodystrophy in 6 patients (10\%), biotin-thiamine-responsive basal ganglia disease (BTBGD) and Leigh disease each in 4 patients (6.7\%), periventricular leukomalacia (PVL) in 3 patients (5\%), and mitochondrial encephalopathy with lactic acidosis and stroke-like episodes syndrome (MELAS) and non-ketotic hyperglycinemia (NKH) each in 2 patients (3.3\%).

Conclusion: Much attention should be paid to pediatric non-traumatic brain injuries. MRI is a safe modality and should be the first radiological investigation if neurological causes are suggested but should be aided by meticulous clinical evaluation and dedicated laboratory investigations for better characterization and differentiation of various causes of non-traumatic, non-infective brain encephalopathy among NICU/PICU patients. When interpreting MRI, it is essential to have thorough relevant clinical data, gestational age at birth which is prognostic of the pattern of hypoxic-ischemic injury, and the time lag between the onset of HII and the time of performing the MR study.
\end{abstract}

Keywords: Encephalopathy, Metabolic disorders, MRI, White matter disorders, Neonate, Child

* Correspondence: tamirhaq@yahoo.com

Department of Radiology, Faculty of Medicine, Zagazig University, Zagazig,

Egypt

(c) The Author(s). 2020 Open Access This article is licensed under a Creative Commons Attribution 4.0 International License, which permits use, sharing, adaptation, distribution and reproduction in any medium or format, as long as you give appropriate credit to the original author(s) and the source, provide a link to the Creative Commons licence, and indicate if changes were made. The images or other third party material in this article are included in the article's Creative Commons licence, unless indicated otherwise in a credit line to the material. If material is not included in the article's Creative Commons licence and your intended use is not permitted by statutory regulation or exceeds the permitted use, you will need to obtain permission directly from the copyright holder. To view a copy of this licence, visit http://creativecommons.org/licenses/by/4.0/. 


\section{Background}

Causes of encephalopathy in neonates/pediatrics include hypoxic-ischemic injury (which is the most frequent cause and is defined as any impairment to the brain caused by insufficient blood flow and oxygenation), trauma, metabolic disorders, and congenital and infectious diseases. The hypoxic-ischemic encephalopathy term should not be confused with the hypoxic-ischemic injury term as hypoxic-ischemic encephalopathy is diagnosed on the basis of specific clinical findings of intense acidosis, poor Apgar score at birth (0-3), seizure, hypotonia, coma, and multiple organ dysfunction. Hypoxic-ischemic injury causing encephalopathy is usually expressed within the first few hours after birth while if encephalopathy happens later (e.g., few days after birth) and with no clear cause, metabolic and infectious causes should be considered. When analyzing MR images of neonatal encephalopathy, it is essential to have thorough relevant clinical history, gestational age at birth which is prognostic of the pattern of hypoxic-ischemic injury (preterm infants with a gestational age of less than 36 weeks or full-term neonates at later gestational age), and the time lag between the onset of hypoxic-ischemic insult and the time of performing the MR study which also affects the findings, especially those of diffusion-weighted imaging [1].

Periventricular leukomalacia (PVL) possibly represents toxic insult to premyelinating oligodendrocytes caused by cerebral ischemia, cerebral reperfusion, or both. End-stage PVL shows volume reduction of the periventricular white matter as well as the centrum semiovale, with irregularity and passive dilatation of the wall of the ventricles.

Metachromatic leukodystrophy is an autosomal recessive disorder produced by deficiency of arylsulfatase A which is a lysosomal enzyme necessary for the sulfatides' normal metabolism, which are vital elements of the myelin sheath, so the sulfatides accumulate in various tissues, including the peripheral nerves, brain, liver, gallbladder, and kidneys, and this accumulation within the neurons and glial cells causes the distinctive metachromatic reaction. Abnormal decreased level of arylsulfatase $\mathrm{A}$ in the peripheral blood leukocytes and in urine is the usual biochemical diagnosis of the disease $[2,3]$.

Biotin-thiamine-responsive basal ganglia disease (BTBGD), also known as thiamine-responsive encephalopathy type 2 or thiamine metabolism dysfunction syndrome, is a rare autosomal recessive (mutation to the human SLC19A3 gene), correctable, neurometabolic disorder manifested by progressive encephalopathy requiring high level of suspicion to diagnose. The disease is characterized by early onset encephalopathy (usually below the age of 4 years). In many case reports, febrile illness preceded the manifestation disease; this febrile illness progresses to severe disability, dystonia, ataxia, seizures, and death if the patients were not treated with biotin and thiamine supplements [4-7].
Leigh disease (or subacute necrotizing encephalomyelopathy) is a progressive, inherited, neurodegenerative disease of infancy/early childhood showing variable disease course and outcome. Hypotonia and psychomotor deterioration is the typical clinical presentation; moreover, ptosis, ophthalmoplegia, dystonia, ataxia, and difficulty in swallowing usually develop. The pathological characteristic disorder includes vascular proliferation, micro-cystic cavitation, neuronal loss, and demyelination of the basal ganglia, cerebellar dentate nuclei, and midbrain and sometime of the cerebral white matter $[2,3,8]$.

Patients are affected with mitochondrial encephalopathy with lactic acidosis and stroke-like episodes syndrome (MELAS) which is a rare disease illness that usually begins in childhood, usually between 2 and 50 years of age with reported rare early neonatal onset. The disease mostly affects the muscles and the nervous system with recurrent cerebral stroke-like injuries showing progressive course with episodic acute exacerbation [2-4].

Non-ketotic hyperglycinemia $(\mathrm{NKH})$ is a neurometabolic, autosomal recessive disorder characterized by poor cleavage of glycine enzyme. The patients carrying the attenuated form of the disease with at least one mutation with residual enzyme activity are still preserved; those patients can present with equal incidence either in the neonatal period or later in infancy period, show mild to severe delayed development, and may have associated seizures. Patients with the severe form of disease present early in the first week of life typically with myoclonic epilepsy often with EEG changes, lethargy progressing to coma, and apnea that requires ventilation [1, 4, 8-12].

The aim of this study is to evaluate the value of MRI in detection and possible differentiation of different nontraumatic, non-infectious causes of acute neonatal/ pediatric encephalopathy among NICU/PICU patients.

\section{Methods \\ Patients}

This retrospective study was conducted between January 2018 and September 2019. There is a revision of the medical records of 80 patients admitted to neonatal intensive care unit or pediatric insensitive care unit (NICU/PICU) during this period for various neurological causes of admission.

The inclusion criteria were patients with positive brain MRI findings for non-traumatic, non-infectious acute brain injury.

Exclusion criteria were positive lumbar puncture for infectious causes, post-traumatic patients, incomplete MRI study, and poor quality images.

By applying the inclusion criteria; 60 patients were selected (32 girls and 28 boys) and had a mean age of 1.1 \pm 1.02 years (range 3 days -11 years), and all presented with variable neurological symptoms and signs (like 
seizures, acute encephalopathy, dystonia, ataxia, ophthalmoplegia) that necessitated NICU/PICU admission.

In the selected 60 cases, the medical files were reviewed for laboratory investigations, genetic testing if any, and the possible final diagnosis in the discharge summary. As a routine for all ICU admitted patients, trans-cranial US and/or plain CT was done upon admission till the arrangement for MRI was done.

The local ethics committee of our institution ruled that the informed consent from the legal guardian was not needed as this is a retrospective study and all patients' medical information were hidden.

\section{MRI technique}

All selected patients had MRI of the brain using a closed 1.5T MRI machine (Philips, Achieva, Netherlands B.V.) utilizing the standard head coil used for adults with applications of foam pads to centralize the head with the patients examined in a supine position.

Post-contrast acquisitions were done in 4 patients by intravenous injection of magnevist $0.1 \mathrm{mmol} / \mathrm{kg}$ [gadolinium diethylene-triaminepenta-acetic acid (Gd-DTPA); Berlex, Montvale, NJ] after making sure that the renal function test was normal.

Before performing the MR imaging, communication with staff in the NICU/PICU was done to avoid waiting of the patient in the MR unit. The NICU/PICU staff were responsible for the monitoring of the vital signs, and cardiac monitoring was done using pulse oximeter and ECG system; all are compatible with MRI. All exams were done by conscious sedation by giving oral chloral hydrate by the ICU/PICU staff. Sponge ear plugs dedicated for neonates were utilized for noise attenuation.

\section{Image acquisition}

The pulse sequences are as follows: axial FLAIR (TR/TE $4800 / 300 \mathrm{~ms}$ ), axial and sagittal T1-WI fast spin-echo
(TR/TE 600/20-25), axial and coronal T2-WI fast spinecho (TR/TE 1500-3000/70-100 ms), axial T2*-WI (T2FFE "fast field echo") (TR/TE 700/23 ms), and flip angle of $18^{\circ}$.

DWI was performed (TR/TE 6642/97 ms), with $b$ value 0 , and $1000 \mathrm{~s} / \mathrm{mm}^{2}$; the ADC map was automatically generated from the DWI images.

There is a slice thickness of $3-4 \mathrm{~mm}$ and inter-slice gap $=1 \mathrm{~mm}$, matrix size $230 \times 230 \mathrm{~mm}^{2}$, and flip angle of $90^{\circ}$.

\section{Analysis of the MRI images}

Images were reviewed on the PACS workstations (Philips, iSite radiology) by two independent neuroradiologists with more than 10 years' experience in pediatric neuroimaging; both reviewers were blinded to the final diagnosis but were provided with the clinical data documented on the PACS by the referring physician. Each MRI was evaluated for the following:

1- The characteristic of the white matter abnormality and its distribution

2- Is there any restricted diffusion at DWI?

3- Is there any enhancement (if contrast was given)?

4- Any associated congenital abnormality

5- Grading of HII severity

Regarding hypoxic ischemia injury (HII) grading, mild to moderate grade (peripheral pattern) was considered if there was affection of the water shed areas (high T2 and FLAIR signal), with restricted diffusion. Severe grade (basal ganglia-thalamic pattern) was considered if there was an extension of the white matter high T2 and FLAIR signal into the basal ganglia, thalami, and hippocampus and corticospinal tracts with restricted diffusion.

Then, initiation provisional diagnosis was written and compared between both reviewers; controversy between

Table 1 General clinical findings of the selected group patients $(n=60)$

\begin{tabular}{|c|c|c|}
\hline Disorder & Number & Clinical key findings \\
\hline 1. Hypoxic ischemia injury (HII) & $39(65 \%)$ & Varying degree of encephalopathy according to its severity \\
\hline 2. Metachromatic leukodystrophy & $6(10 \%)$ & $\begin{array}{l}\text { Autosomal recessive (AR), motor signs of acute peripheral neuropathy, deterioration } \\
\text { in intellect, speech, and coordination, gait disturbance, quadriplegia in } 2 \text {, and blindness } \\
\text { in } 1 \text { patient. Death occurred in } 2 \text { patients of them after } 6 \text { months of initial admission. }\end{array}$ \\
\hline 3. BTBGD & $4(6.7 \%)$ & $\begin{array}{l}\text { AR, early onset encephalopathy (before the age of } 4 \text { years), and febrile illness progress } \\
\text { to acute encephalopathy, dystonia, ataxia, and seizures and death if the patients were } \\
\text { not treated with biotin and thiamine supplements }\end{array}$ \\
\hline 4. Leigh disease & $4(6.7 \%)$ & Hypotonia, ataxia, ptosis, ophthalmoplegia, and dystonia \\
\hline 5. Periventricular leukomalacia (PVL) & $3(5 \%)$ & Premature (33-36 weeks of gestation) all showed delayed milestones \\
\hline 6. MELAS & $2(3.3 \%)$ & $\begin{array}{l}\text { Healthy at birth with early normal development, then growth delay, seizures, episodic } \\
\text { vomiting, and recurrent cerebral stroke-like insult }\end{array}$ \\
\hline 7. Non-ketotic hyperglycinemia & $2(3.3 \%)$ & Acute encephalopathy shortly after delivery \\
\hline Total & 60 & \\
\hline
\end{tabular}


the two observers was solved by consensus after a conjoint evaluation of the MRI images. Then, the final diagnosis of both reviewers was compared with the final diagnosis in the discharge based on MRI suggestive findings, laboratory-specific findings, genetic testing (if any), clinical data findings, and follow-up in mitochondrial encephalopathy with lactic acidosis and stroke-like episodes syndrome (in case of new stroke) or after specific treatment in cases of biotin-thiamine-responsive basal ganglia disease (after 4-6 months after treatment).

\section{Statistical analysis}

The data were statistically analyzed after data collection and tabulation using Statistical Package for the Social Sciences (SPSS) version 21. Descriptive statistic was performed in a form of number and percentage for qualitative data.

The inter-observer agreement was calculated. The percentage of agreement statistic as well as the kappa statistic was used to calculate the percent of agreement above the expected by chance.
Agreement was considered moderate when there is a weighted kappa statistic of $0.41-0.60$, good when it is $0.61-0.80$, and excellent when it is $0.81-1.00$, as defined by Fagon et al. [13].

\section{Results}

This retrospective study included 60 selected patients according to the study inclusion and exclusion criteria, and all presented with positive MRI findings for nontraumatic, non-infectious acute brain injury. Females (32, 53.3\%) were affected more than males (28, 46.7\%) with a mean age of $1.1 \pm 1.02$ years (range 3 days-11 years), and all presented with variable neurological symptoms and signs that necessitate NICU/PICU admission [summary of clinical presentation in Table 1].

The final diagnosis of the study group patients was hypoxic ischemia injury (HII) in 39 patients (65\%, Figs. 1 and 2), metachromatic leukodystrophy in 6 patients (10\%, Figs. 3, $4)$, BTBGD and Leigh disease each in 4 patients (6.7\%, Figs. 5 and 6), PVL in 3 patients (5\%, Fig. 7), and MELAS and NKH each in 2 patients (3.3\%, Figs. 8 and 9) [Table 2].
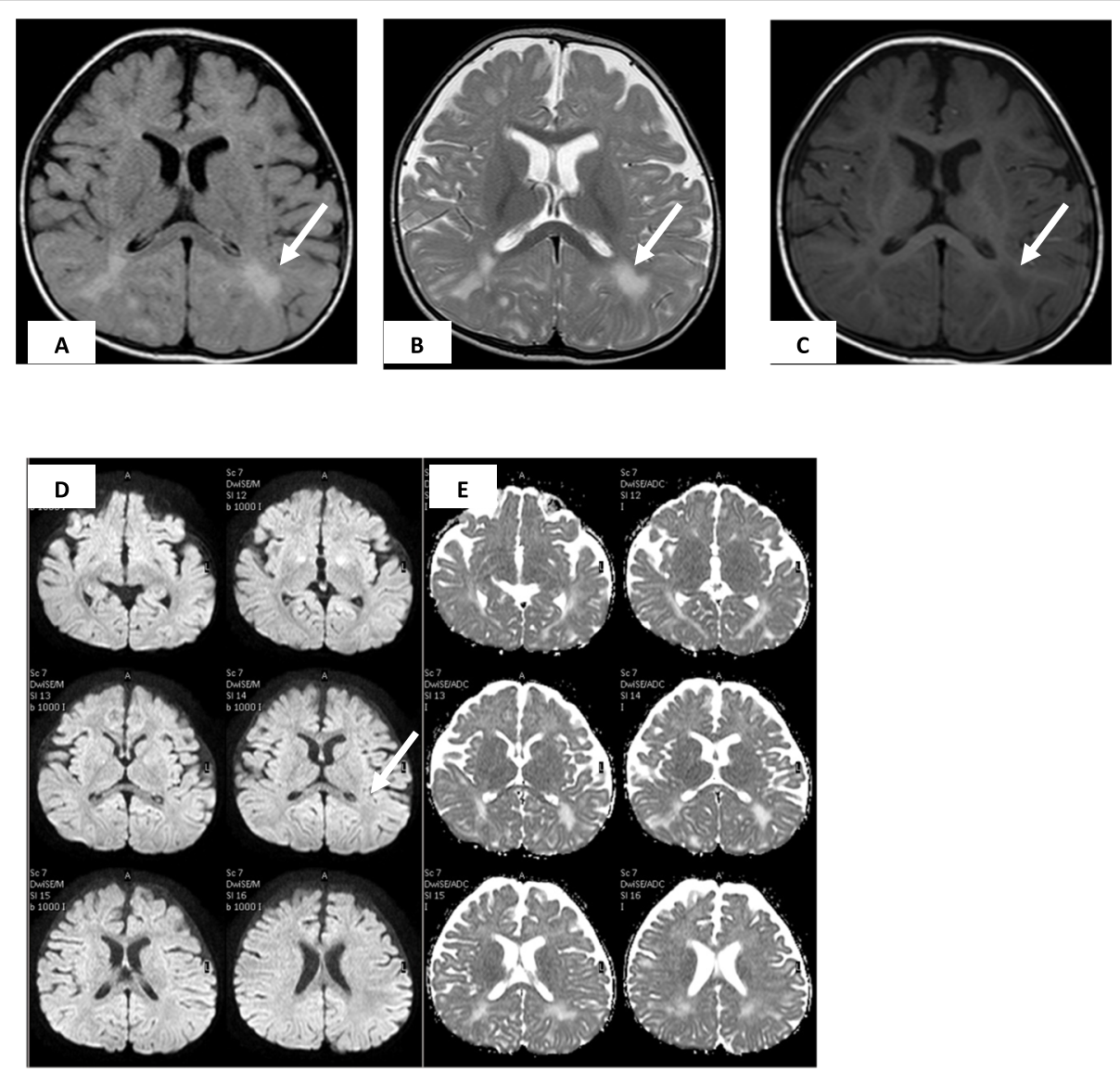

Fig. 1 Nine-month-old girl, PICU admitted for prolonged asphyxia with diagnosis of mild HII. MRI done 8 days after admission showing bilateral symmetrical areas of abnormal signal involving the juxta-cortical, subcortical and periventricular white matter (white arrows). They elicit low/ intermediate signal on T1 (C) and high signal on T2 (B) and FLAIR (A) with no restricted DWI (D,E) "pseudonormalization" although neurologically unstable. 

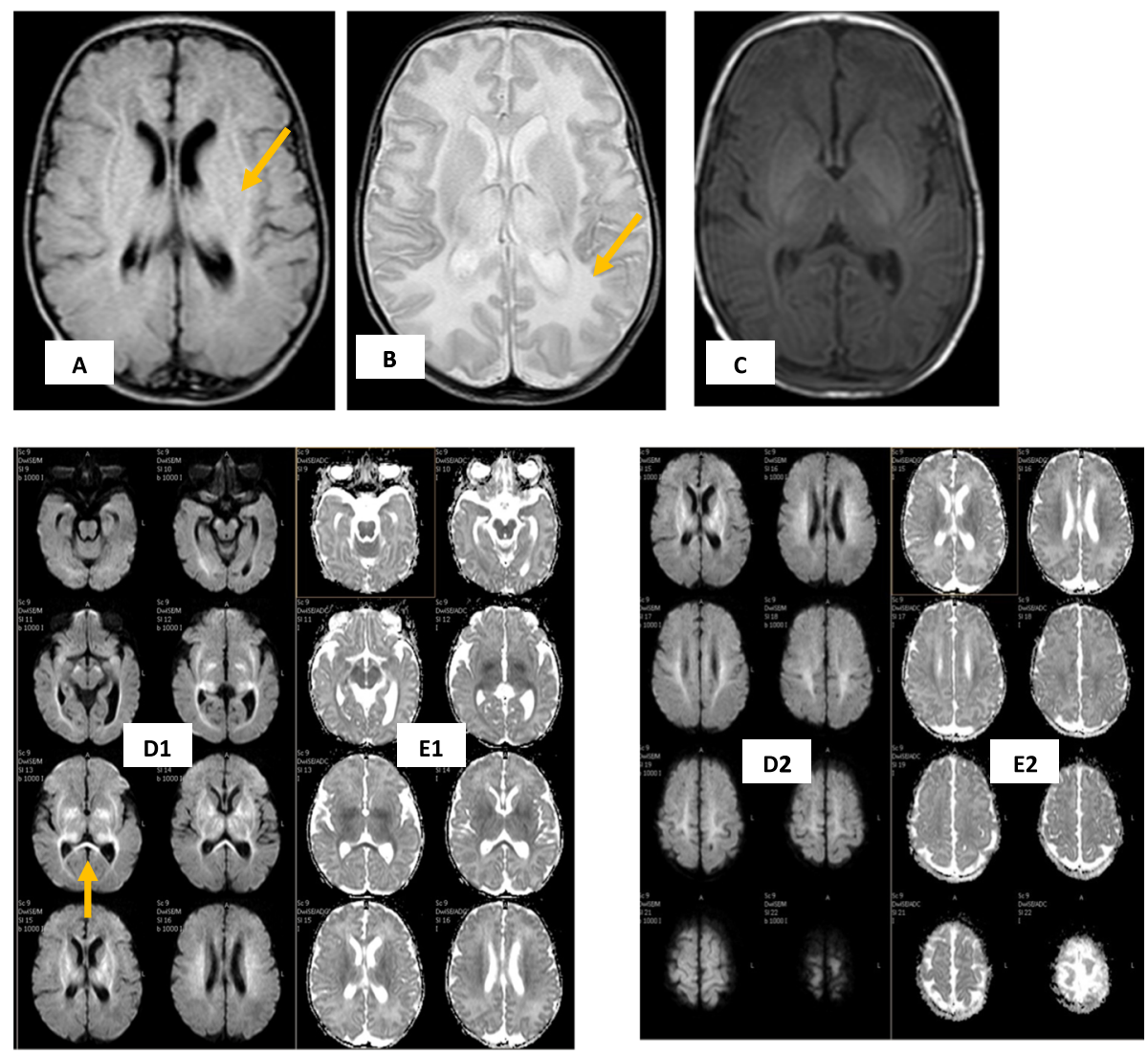

Fig. 2 3-months-old girl with sever HII. Different sequences MRI showed high FLAIR signal (yellow arrow in $\mathbf{A}$ ) at both BG, thalami as well as the white matter (yellow arrow in B) extending to the corpus callosum (yellow arrow in D1), hippocampus and corticospinal tracts (basal gangliathalamic pattern). DWI showed restricted diffusion in acute phase.

Regarding hypoxic ischemia injury (HII) grading, 30 cases $(76.9 \%)$ were mild to moderate grade while 9 cases (23.1\%) were severe grade [Table 3]. In the mild to moderate grade group $(n=30)$, MRI revealed affection of the water shed areas in all cases, showing high T2 and FLAIR signal. DWI showed restricted diffusion in 29 cases while 1 case (2.5\%) of post-chocking with resultant prolonged asphyxia showed free diffusion in MRI. There was delay in performing MRI in this patient due to bad general condition of the patient, so MRI was done 8 days after admission (Fig. 1).

In the severe grade cases of HII, MRI showed extension of the white matter high T2 and FLAIR signal into the basal ganglia, thalami, and hippocampus and corticospinal tracts with restricted diffusion in all cases [Table 3, Fig. 2].

Regarding the 6 patients (10\%) of metachromatic leukodystrophy, all patients were infantile in form as their age of presentation was 11-19 months, and 3 of them gave family history of death in brother/sister at the same age. Clinical presentations were motor signs of acute peripheral neuropathy, speech, gait and coordination disturbance, intellect deterioration, quadriplegia in 2, and blindness in 1 patient. Death occurred in 2 of them after 6 months of initial admission. Laboratory investigations revealed low levels of arylsulfatase A in urine and in peripheral blood leukocytes.

MRI revealed bilateral symmetrical confluent foci of high T2 and FLAIR signal intensity in the periventricular white matter with sparing of the subcortical U-fibers (Fig. 3) with no enhancement at contrast series (Fig. 4). The periventricular white matter and centrum semiovale showed tigroid patterns of demyelination, suggesting sparing of the perivascular white matter. Extension of the abnormality into the corpus callosum and the internal capsule as well as corticospinal tracts also were noted.

Regarding the 4 patients of BTBGD (6.7\%, Fig. 5), the age of the patients ranged from 2 to 4 years, all with positive consanguinity and positive family history, manifested by febrile illness progressing to acute encephalopathy which ultimately progressed to dystonia, ataxia, and seizures. MRI revealed bilateral high T2 and FLAIR signal affecting the basal ganglia and thalami as well as the white matter with diffusion restriction. The brain stem 

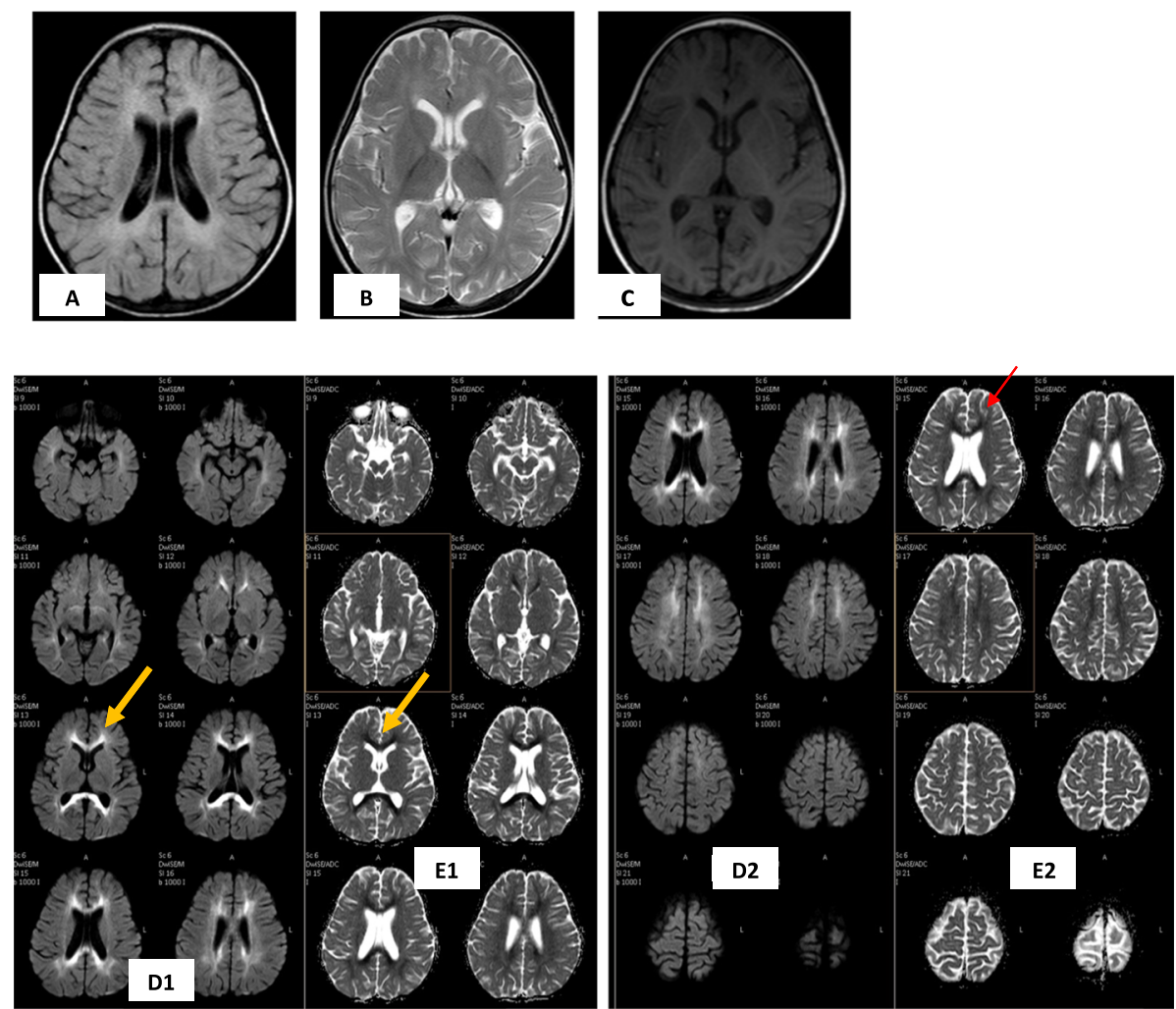

Fig. 3 19-month-old boy with infantile form metachromatic leukodystrophy, positive family history of children death at the age of 20 months. Different sequences MRI revealed bilateral symmetrical areas of high T2 and FLAIR signal in the periventricular white matter with sparing of the subcortical $U$ fibers (red arrow in E2) and extension into the corpus callosum (yellow arrow in D1).

and cerebellum were relatively spared. The patients were started on pulse steroid therapy $(20 \mathrm{mg} / \mathrm{kg} /$ day) for 5 days but showed no improvement. The MRI finding, lack of clinical improvement, and the clinical suspicion suggested the suspicion of biotin-thiamine-responsive basal ganglia disease, so the empirical treatment was started and the patient showed marked improvement. The follow-up MRI after 1-6 months showed resolved lesions after treatment. Genetic testing revealed mutation of the SLC19A gene which confirmed the diagnosis.

Regarding the 4 patients of Leigh disease (6.7\%, Fig. 6), all patients had positive family history, presented with hypotonia, ataxia, ptosis, ophthalmoplegia, and dystonia. MR showed high T2WI and FLAIR signal at both BG, thalami, dorsal brainstem, and the white matter. DWI showed restricted diffusion in acute phase.

In respect to the 3 patients (5\%) of PVL, all of them were premature (33-36 weeks of gestation), 2 of them showed cerebral cyst with hematoma within at antenatal US, and all showed delayed milestones. MRI revealed intra-axial cystic lesions with small hemorrhagic components within (high T1 and T2 signal with minimal blooming at $\mathrm{T} 2 *$ ) in 2 patients, abnormal periventricular white matter signal displaying high signal at $\mathrm{T} 2$, and FLAIR with no restricted diffusion as well as markedly thinned corpus callosum.

In the 2 patients (3.3\%, Fig. 8) with MELAS syndrome, the age of presentations was 4 months and 5 years, and both presented with recurrent vomiting, seizures, and recurrent cerebral strokes. Laboratory investigations revealed elevated serum lactate level. MR showed multiple cortical and subcortical infarct-like lesions extending to the basal ganglia and both thalami and cerebral hematoma, together with a variable degree of generalized cerebellar and cerebral atrophy. DWI showed marked restricted diffusion at the previous sites, and $\mathrm{T}^{*}$ showed minimal blooming at the site of the hematoma. Follow-up MR showed resolution and newly developed similar foci. The final diagnosis was reached by muscle biopsy that showed ragged red fibers.

The 2 patients of non-ketotic hyperglycinemia (3.3\%, Fig. 9) presented shortly after delivery by acute encephalopathy with neurologic impairment. MRI revealed mildly dilated ventricular system with corpus callosum agenesis as well as high T2 and FLIAR signal of the deep white matter with diffusion restriction at DWI. 

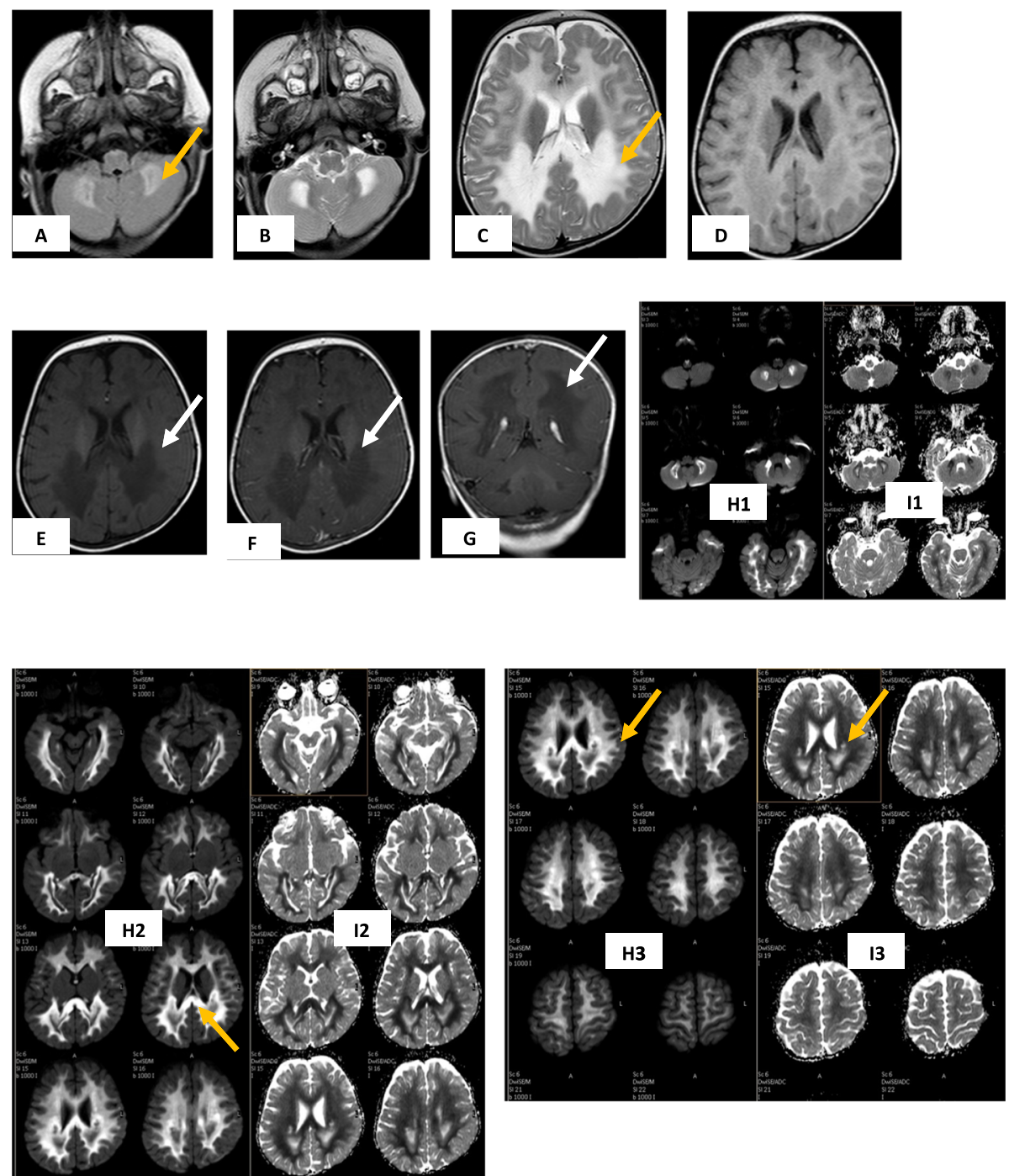

Fig. 4 15-yers-old girl with infantile form metachromatic leuckdystrophy. Different sequences MRI revealed bilateral symmetrical confluent foci of high T2 and FLAIR signal in the periventricular white matter (yellow arrows) with no enhancement at contrast series (white arrows in $\mathbf{F}, \mathbf{G}$ ). The periventricular white matter and centrum semiovale showed tigroid patterns of demyelination (yellow arrow in " $\mathbf{C}^{\prime \prime} \mathrm{T} 2 \mathrm{Wl}$ ). Extension of the abnormality into the corpus callosum (yellow arrow in $\mathbf{H 2}$ ) and the internal capsule as well as corticospinal tracts also were noted.

In the present study, the inter-observer agreement was 0.82 using weighted kappa statistics denoting excellent agreement.

\section{Discussion}

Non-traumatic brain injuries (nTBI) can result in longterm adverse consequences and should be carefully recognized. The most common causes of nTBI include hypoxic insult, vascular insults, toxicity, tumors, infective, and metabolic encephalopathy. The impact of nTBI on the health service among adults is currently available, and it was found that the direct cost of healthcare services for nTBI was higher than that of TBI. The impacts of nTBI among the pediatric population are important to study because they are often overlooked with regard to the potential for long-lasting consequences with its need for prolonged healthcare services post-injury [14, 15]. The current study included patients with nontraumatic, non-infectious brain insult that necessitated NICU/PICU admission owing to the importance to study such group of patients in agreement with the previous study [14].

The leading cause of cerebral palsy and death among children is the hypoxic-ischemic injury occurring in about 2-9 of 1000 live births. HII is more common among preterm infants occurring in $5 \%$ of infants born below 32 weeks of gestation. Reduced cerebral blood oxygenation (hypoxemia) and cerebral blood flow 

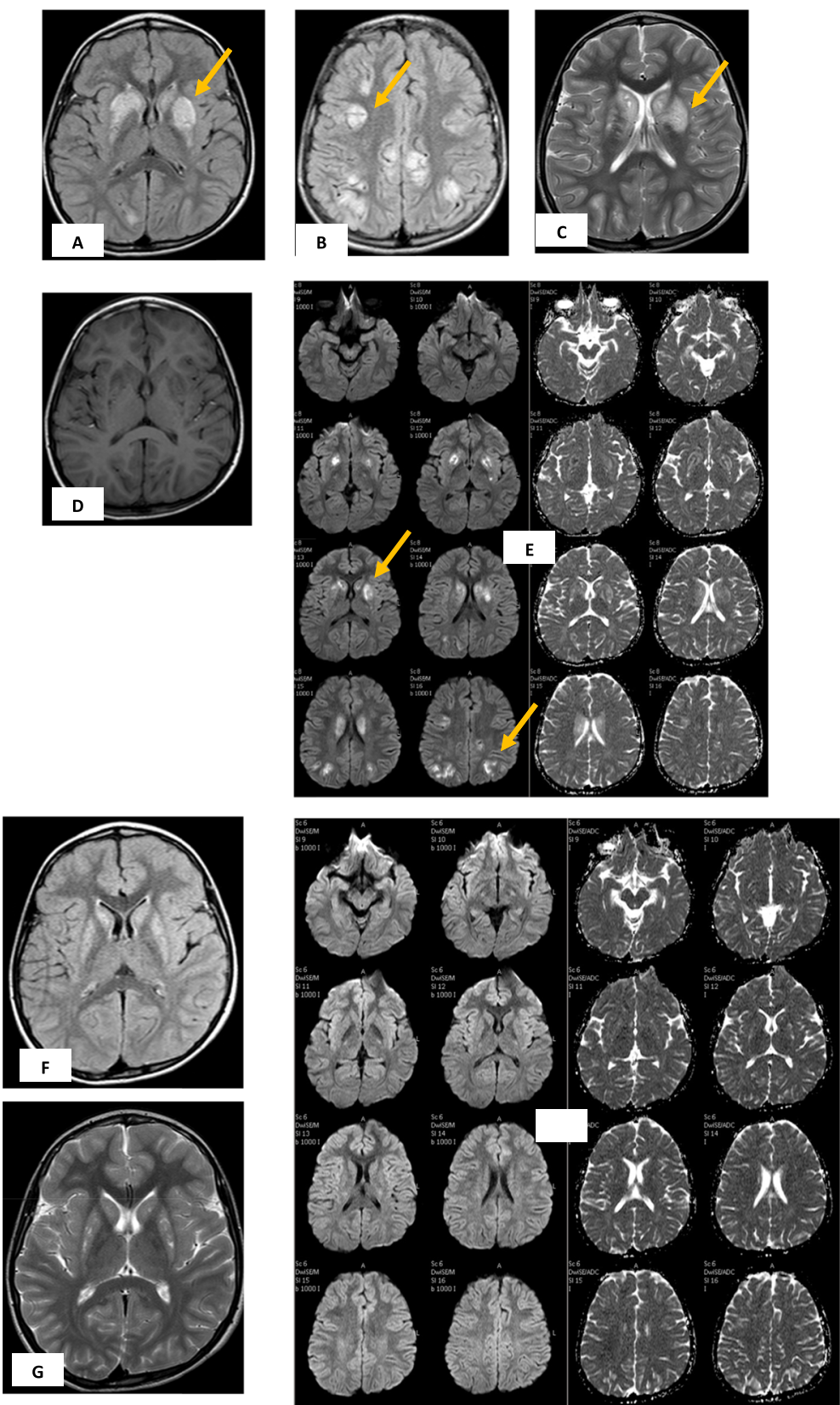

Fig. 5 3-year-old male with BTBGD. Different sequences Initial MRI (March 2018, A-E) showed bilateral high T2 and FLAIR signal affecting the basal ganglia and thalami as well as the white matter with diffusion restriction (white arrows) sparing the brain stem and the cerebellum. Follow up MRI $(\mathbf{F}, \mathbf{G}, \mathbf{H})$ at Sept. 2018 after treatment showed complete resolution.

(ischemia) are the frequent pathophysiologic processes resulting in HII [16].

Mild to moderate HII in the premature brain may lead to germinal matrix hemorrhage, PVL, or both, while severe HII to the premature brain usually causes injury to the thalamus, dorsal brainstem, and anterior part of the vermis with occasional extension to the basal ganglia, cerebellum, hippocampus, and corticospinal tracts. The basal ganglia injury is frequently less severe than thalamic injury [16].

Depending on the severity and the duration of the HII, variable clinical and imaging findings are seen. Mild episodes of hypoxia or ischemia for short duration $(<10 \mathrm{~min})$ are common in the perinatal setting and frequently have no major neurologic consequences. However, HII with greater severity and longer duration are usually associated 

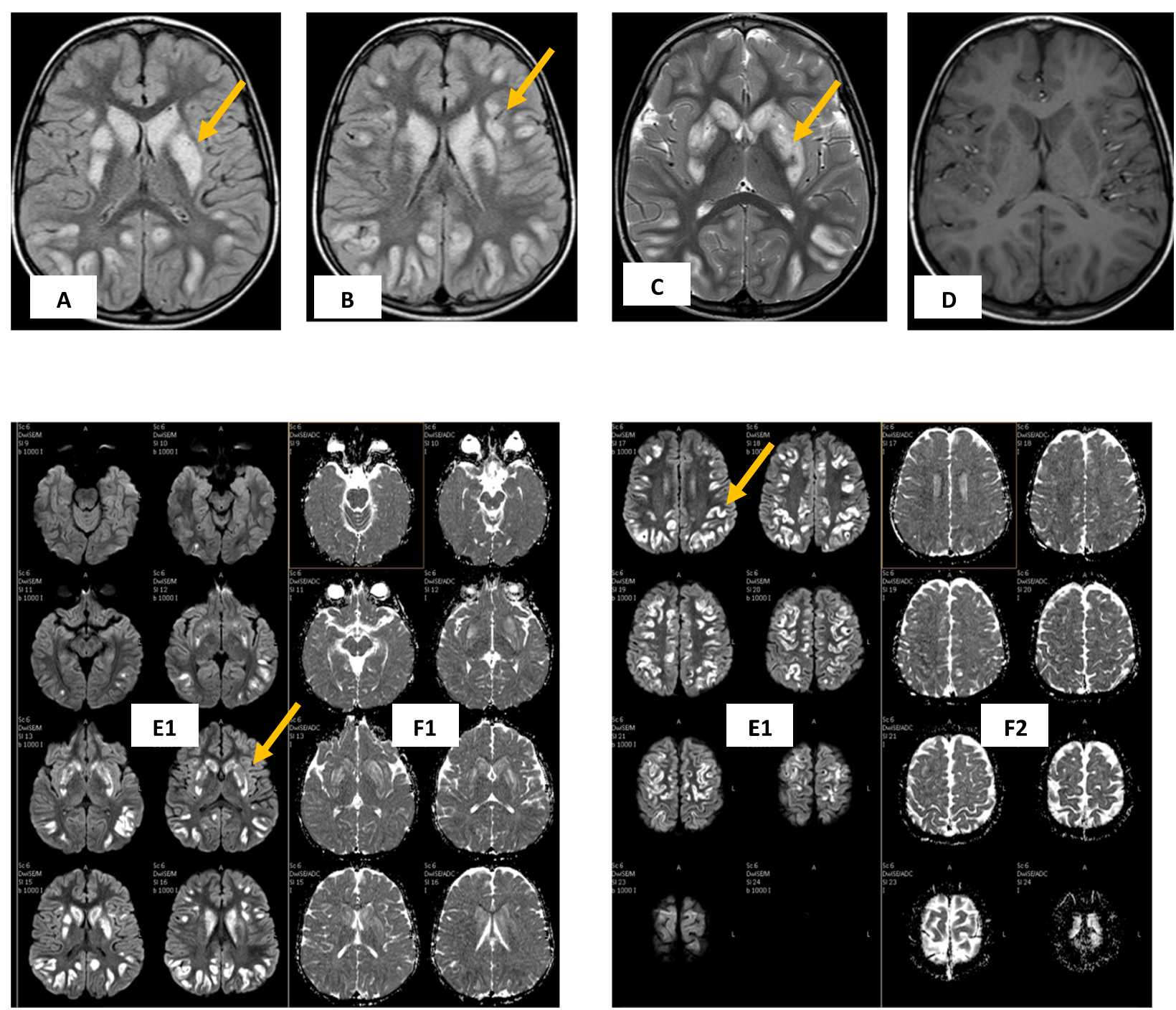

Fig. 6 3-years-old boy with Leigh syndrome. Different sequences MRI revealed high T2WI and FLAIR signal at both BG, thalami and the white matter. DWI showed restricted diffusion in acute phase (yellow arrows).

with clinical and imaging drawbacks. The peripheral pattern (or the parasagittal, watershed, or border zone pattern) is the most frequent pattern and caused by partial hypoxia with prolonged duration or mild to moderate hypotension. The basal ganglia-thalamic pattern is less frequent and caused by severe hypotensive or severe hypoxic insult within shorter duration. Overlap between both patterns is frequently seen. When the insult is severe and of longer duration, the resultant injury is more grave, with involvement of the whole cerebral cortex and dorsal brainstem and resulting in total brain injury pattern $[16,17]$.

This retrospective study included 60 selected patients according to the study inclusion and exclusion criteria. The final diagnosis revealed that hypoxic ischemia injury (HII) was the most common encountered cause found in 65\% in agreement with Manohar et al. [1] and Sonia et al. [16] who mentioned that hypoxic-ischemic injury is the most common cause of neonatal encephalopathy.

Mild to moderate grades of HII (peripheral pattern) was found in $76.9 \%$ of patients with HII, while $23.1 \%$ of the patients were severe grade in agreement with Sonia et al. [16] who mentioned that the peripheral pattern is the most common pattern.

In the mild to moderate grade cases $(n=30)$, the MRI revealed peripheral ischemic pattern (water shed areas) in all cases, displaying high T2 and FLAIR signal in agreement with Sonia et al. [16] who mentioned that the peripheral pattern of HII is frequently seen in the cerebral cortex and subcortical white matter, with 

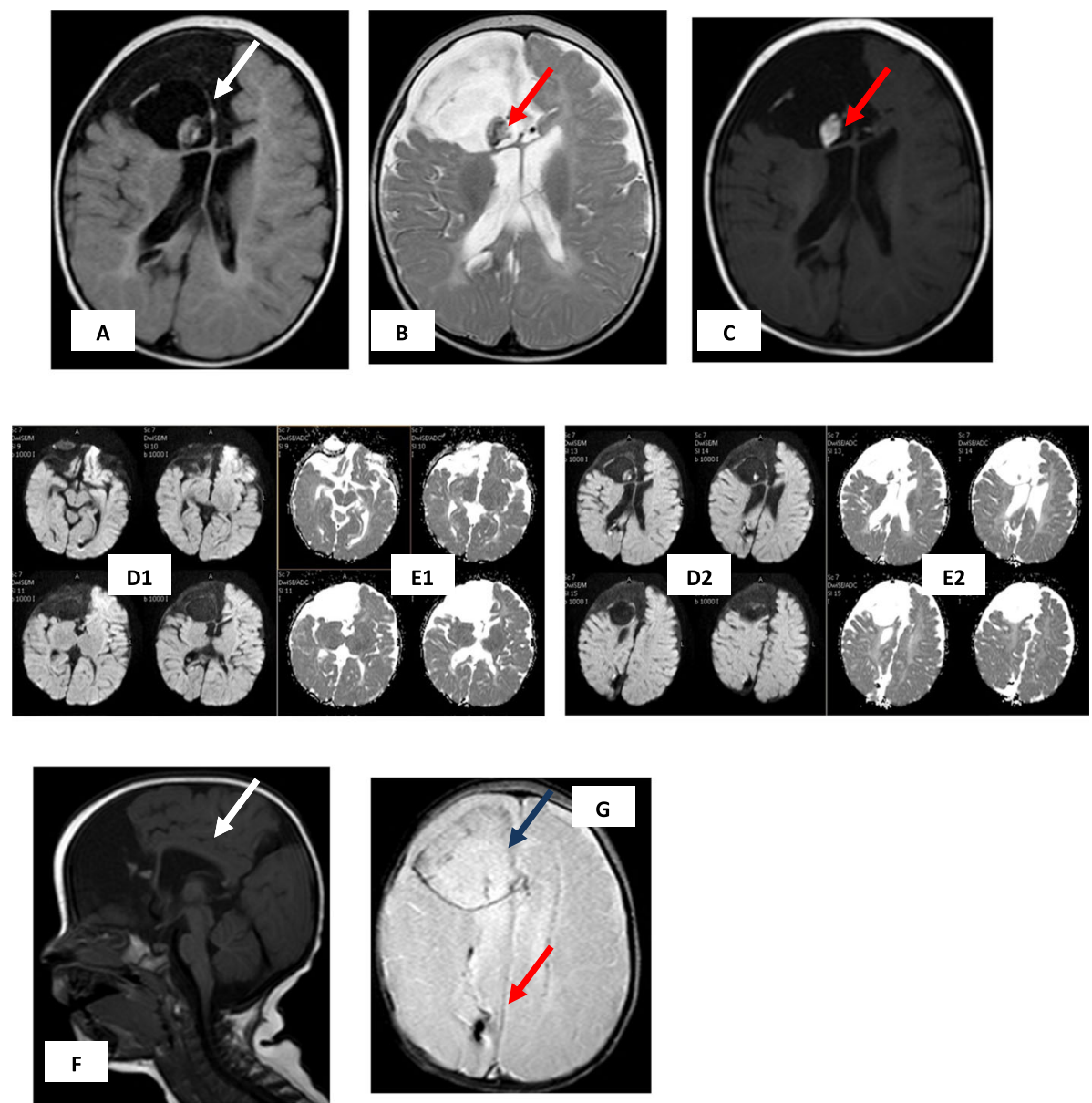

Fig. 7 24-days-old premature girl (33 weeks of gestation), with PVL. MRI reveals right frontal cystic lesion (white arrow in A) compressing the frontal horn of the right lateral ventricle, with intra-cystic small hemorrhagic component (red arrow) displaying high T1 and low T2 signal with minimal blooming at $\mathrm{T} 2 *$ (blue arrow in $\mathbf{G})$. Another hemorrhagic foci at the right posterior parietal region (red arrow in $\mathbf{G}$ ). Abnormal periventricular white matter signal intensity displaying mild high signal at FLAIR and T2WI with no restricted diffusion. Markedly thinned corpus callosum (white arrow in $\mathbf{F}$ ).

predilection to the parieto-occipital and posterior temporal regions more injured than the anterior regions with corresponding restricted diffusion in the acute phase.

In the current study, severe HII showed extension of the white matter high T2 and FLAIR signal lesions into the basal ganglia, thalami, hippocampus, and corticospinal tracts, and all cases showed restricted diffusion, in agreement with Sonia et al. [16] who mentioned that the basal ganglia-thalamic pattern is less common and usually seen in the case of severe hypoxic or severe hypotensive insult within a short duration.

Restricted diffusion was found in DWI in all cases of HII, while one case post-chocking with resultant prolonged asphyxia showed free diffusion, and this could be explained by the delay in performing MRI due to bad general condition of the patient, so MRI was done 8 days after the incident revealing free diffusion (pseudonormalization) in agreement with Manohar et al. [1] and Sonia et al. [16] who mentioned that DWI became falsely negative about 1 week after the ischemic or hypoxic event; this phenomenon is known as pseudonormalization; so, it is vital to know the time of the onset of encephalopathy.

The present study included 6 patients $(10 \%)$ with a final diagnosis of metachromatic leukodystrophy; all patients were infantile in form, and 3 of them had a family history of death in brother/sister at the same early age. Laboratory investigation revealed low levels of arylsulfatase A in urine and in peripheral blood leukocytes in accordance with Cheon et al. [2], Mohannad et al. [3], and Karimzadeh [4] studies who reported that metachromatic leukodystrophy has 3 different types according to the age of the patient at the onset which are late infantile, juvenile form, and adult form, and the most frequent type is late infantile.

The clinical presentations of this group of patients in the current study were motor signs of acute peripheral 

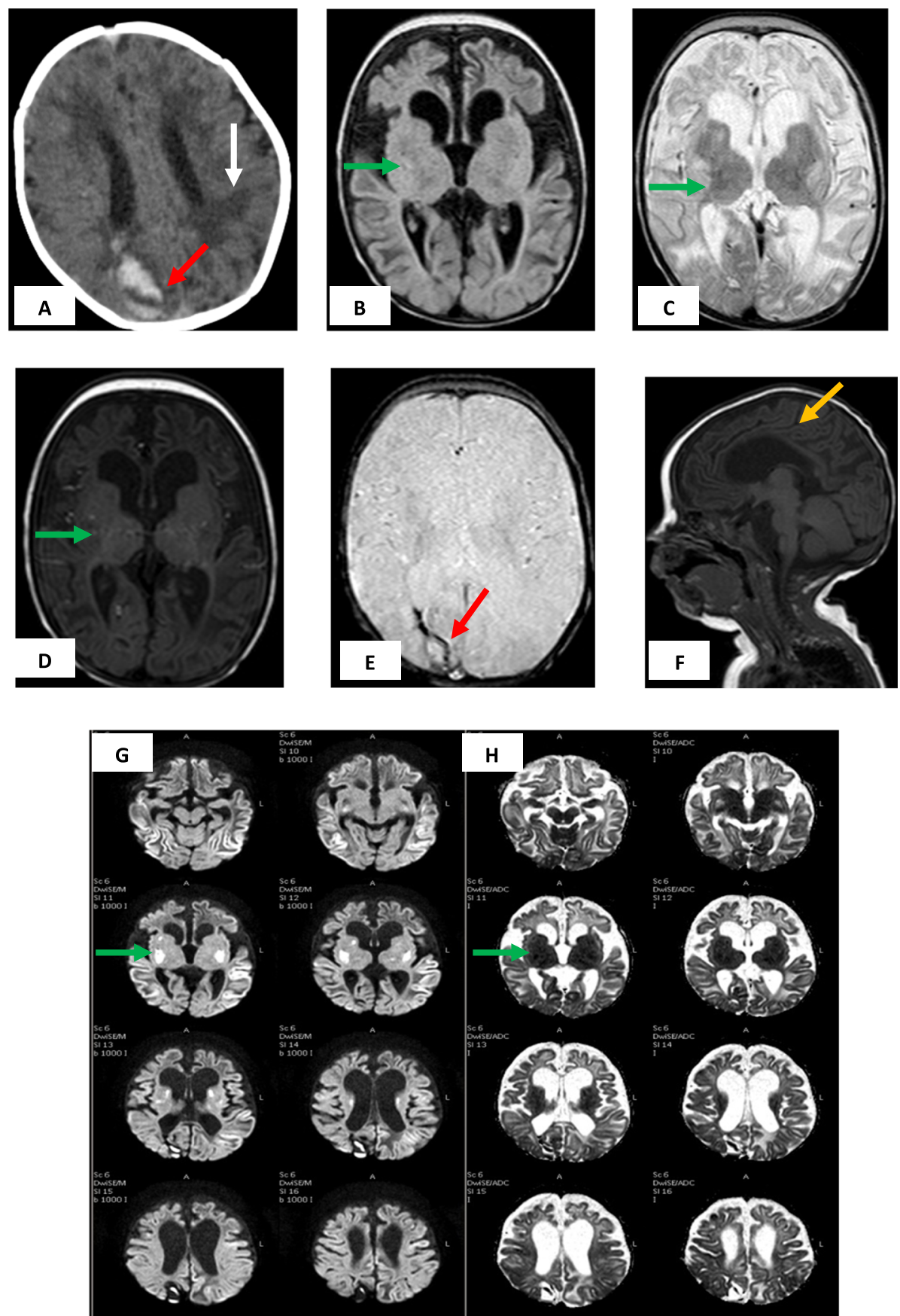

Fig. 84 month-old male with MELAS [A] Non-contrast CT showing periventriculer hypodensities (white arrow) and right occipital hematoma (red arrow). Different sequences axial MRI, [B] FLAIR, [C] T2WI, [D] T1WI, showing isointense signal of the basal ganglia (green arrow), thalami and tempro-parietal cortical gyri at T1 and FLAIR, low signal at T2 with dilated ventricular system. [E] Axial T2* showed minimal blooming (red arrow), [F] sagittal T1 showing markedly thinned corpus callosum (yellow arrow) [G] DWI b1000, [H] ADC map showed restricted diffusion at the previous sites (green arrows)

neuropathy, speech, gait and coordination disturbance, intellect deterioration, quadriplegia in 2 patients, and blindness in 1 patient. Death occurred in 2 of them after 6 months of initial admission in agreement with Cheon et al. [2] who mentioned that the late infantile form of metachromatic leukodystrophy usually manifested in children between the ages of 12 and 18 months and is manifested by peripheral neuropathy motor signs usually 

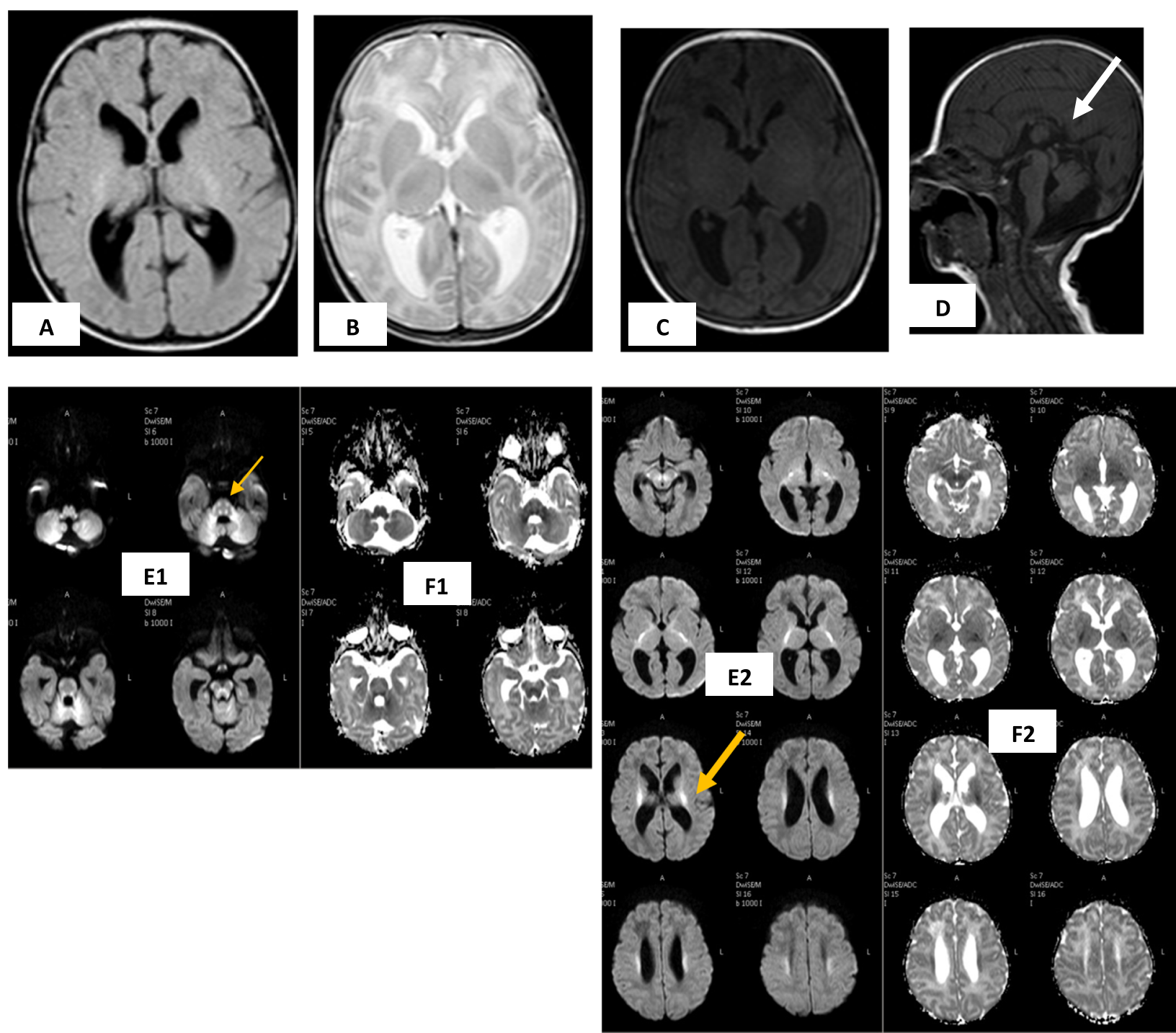

Fig. 9 20-days- old boy with NKH. MRI showing bilateral symmetrical areas along the course of the corticospinal tracts eliciting low signal at T1 (C), high at T2 (B) and FLAIR (A) with restricted diffusion (yellow arrow in E2) and small foci of restricted diffusion along the posterior aspect of the brain stem (yellow arrow in E1), middle cerebellar peduncles and the superior aspect of both cerebellar hemispheres (yellow arrow in E2) associated with corpus callosum hypogenesis (white arrow in $\mathbf{D}$ ).

followed by coordination and speech deterioration. Gait disturbance, blindness, quadriplegia, and decerebrate posturing may be seen within 2 years. The disease may progress, and death occurs within 6 months to 4 years after the onset of disease.

In the current study, MRI of metachromatic leukodystrophy patients revealed bilateral symmetrical confluent foci of high T2 and FLAIR signal intensity in the periventricular white matter with sparing of the subcortical Ufibers with no enhancement. The periventricular white matter and centrum semiovale showed tigroid patterns of demyelination, suggesting sparing of the perivascular white matter. Extension of the abnormality into the corpus callosum and the internal capsule as well as corticospinal tracts also were noted. This was in concordance with Cheon et al. [2], Mohannad et al. [3] and Karimzadeh [4] studies who reported similar imaging findings.

The current study included 4 patients (6.7\%), with a final diagnosis of BTBGD; the age of the patients ranged from 2 to 4 years, all with positive consanguinity and positive family history, manifested by febrile illness progressing to acute encephalopathy which ultimately progressed to dystonia, ataxia, and seizures in agreement with Alabdulqader et al. [5] who mentioned a similar clinical presentation.

MRI reveals high signal at T2 and FLAIR affecting both basal ganglia and thalami as well as the white 
Table 2 General MRI findings of the selected group patients $(n=60)$

\begin{tabular}{|c|c|c|c|}
\hline Disorder & Number & Conventional MRI findings & DWI \\
\hline $\begin{array}{l}\text { 1. Hypoxic ischemia injury } \\
\text { (HII) }\end{array}$ & $39(65 \%)$ & According to the severity & $\begin{array}{l}\text { Restricted diffusion in affected areas } \\
\text { in acute phase }\end{array}$ \\
\hline $\begin{array}{l}\text { 2. Metachromatic } \\
\text { leukodystrophy }\end{array}$ & $6(10 \%)$ & $\begin{array}{l}\text { High T2/FLAIR signal in the deep white matter sparing } \\
\text { the U-fibers with tigroid pattern }\end{array}$ & $\begin{array}{l}\text { Restricted diffusion in affected white } \\
\text { matter }\end{array}$ \\
\hline 3. BTBGD & $4(6.7 \%)$ & $\begin{array}{l}\text { High T2/FLAIR signal in both basal ganglia, thalami, and } \\
\text { the white matter becomes resolved after treatment }\end{array}$ & Restricted diffusion in acute phase \\
\hline 4. Leigh disease & $4(6.7 \%)$ & $\begin{array}{l}\text { High T2/FLAIR signal in the basal ganglia, thalami, brainstem, } \\
\text { and the white matter }\end{array}$ & Restricted diffusion in acute phase \\
\hline $\begin{array}{l}\text { 5. Periventricular } \\
\text { leukomalacia (PVL) }\end{array}$ & $3(5 \%)$ & $\begin{array}{l}\text { High T2 periventricular foci, minimal blooming at T2* for } \\
\text { hemorrhage }\end{array}$ & $\begin{array}{l}\text { Early restricted diffusion with } \\
\text { normalization within } 5-7 \text { days }\end{array}$ \\
\hline 6. MELAS & $2(3.3 \%)$ & $\begin{array}{l}\text { Multiple subcortical and cortical infarct-like lesions, with variable } \\
\text { degrees of generalized cerebellar and cerebral atrophy with } \\
\text { frequent involvement of the parietal, occipital, and the basal ganglia. }\end{array}$ & Restricted diffusion in acute phase \\
\hline $\begin{array}{l}\text { 7. Non-ketotic } \\
\text { hyperglycinemia }\end{array}$ & $2(3.3 \%)$ & $\begin{array}{l}\text { Diffuse high T2 and FLAIR signal of the white matter and the } \\
\text { cerebral cortex }\end{array}$ & Restricted diffusion in acute phase \\
\hline
\end{tabular}

matter with diffusion restriction. The brain stem and cerebellum were relatively spared. The follow-up MRI after 1-6 months showed resolved lesions after treatment. Genetic testing in those patients revealed mutation of the SLC19A gene in agreement with Karimzadeh [4], Alabdulqader et al. [5], and Sremba et al. [7] who reported similar MRI imaging findings and genetic testing results and concluded that the typical MRI finding, lack of clinical improvement, and the clinical suspicion should raise the possibility of BTBGD, so empirical treatment should started.

This study included 4 patients (6.7\%) with Leigh disease; all patients had positive family history and presented with hypotonia, ataxia, ptosis, ophthalmoplegia, and dystonia in agreement with Cheon et al. [2] and Mohannad et al. [3] who reported that Leigh disease is a progressive, inherited, neurodegenerative disease of infancy/early childhood with variable disease course and prognosis. Affected infants/children frequently presented with psychomotor deterioration, hypotonia, ophthalmoplegia, dystonia, ataxia, ptosis, and difficulty in swallowing.

MRI revealed high T2WI and FLAIR signal at both basal ganglia, thalami, dorsal brainstem, and the white matter with diffusion restriction in acute phase in agreement with Cheon et al. [2] and Mohannad et al. [3] who reported that the typical MR imaging findings include bilateral symmetric involvement of the putamen and may be associated with abnormalities in the caudate nuclei, globus pallidi, brainstem, thalami, and less frequently the cerebral cortex with rare affection of the cerebral white matter.

The present study included 3 patients (5\%) with MRI diagnosis of PVL; all of them were premature (33-36 weeks) and showed delayed milestones, 2 of them showed cerebral cyst with small hematoma within at ante-natal US, and all presented with delayed milestones. MRI revealed intra-axial cystic lesions with small hemorrhagic component within (high T1 and T2 signal with minimal blooming at $\mathrm{T}_{2} *$ in 2 patients and abnormal periventricular white matter signal displaying high signal at T2 and FLAIR with no restricted diffusion as well as markedly thinned corpus callosum. This was in consistent with Manohar et al. [1] who reported that PVL has two variants, the cystic one which is characterized by areas of focal necrosis at the deep white matter or non-cystic variant which shows more diffuse insult to the premyelinating oligodendrocytes. The lesions in PVL do not show or show minimal blooming at $\mathrm{T} 2 *$ susceptibility-weighted sequence, unlike hemorrhagic lesions. DWI show early diffusion restriction and normalize within 5-7 days. Corpus callosum thinning especially its posterior body and splenium is a frequently associated feature.

This study included 2 patients $(3.3 \%)$ with a final diagnosis of MELAS, presented with recurrent vomiting, seizures, and recurrent cerebral strokes. Laboratory investigations revealed elevated serum lactate levels in agreement with Cheon et al. [2] and Karimzadeh et al. [4] who

Table 3 Grades of HII and their DWI findings

\begin{tabular}{llll}
\hline Grade & Site of affection & Number & DWl in acute stage \\
\hline Mild to moderate & Water shed, white matter & $30(76.9 \%)$ & $-29(74.4 \%)$ showed restricted DWl \\
Severe & Extends to involve BG and thalami & $9(23.1 \%)$ & $-9(23.1 \%)$ showed restricted DWI \\
\hline
\end{tabular}


mentioned that MELAS syndrome patients are usually seen healthy at birth with early normal development, then show growth delay, seizures, episodic vomiting, and recurrent cerebral stroke-like injuries. Cerebrospinal fluid and serum lactate levels are frequently usually elevated.

MRI of this group in the current study showed multiple cortical and subcortical infarct-like lesions extending to the basal ganglia and both thalami and cerebral hematoma, together with a variable degree of generalized cerebellar and cerebral atrophy. DWI showed marked restricted diffusion at the previous sites, and $\mathrm{T}^{*}$ showed minimal blooming at the site of the hematoma. The follow-up MR showed resolution and newly developed similar foci in agreement with Cheon et al. [2], Mohannad et al. [3], and Karimzadeh [4] who reported similar imaging findings.

Current study included 2 patients (3.3\%) with final diagnosis of non-ketotic hyperglycinemia presented shortly after delivery by acute encephalopathy with neurologic impairment in agreement with Manohar et al. [1] and Mohammad [10] who reported that NKH is an autosomal recessive neurometabolic disease causing neurologic impairment usually evident soon after delivery.

The MR imaging findings of this group in the current study included mildly dilated ventricular system with corpus callosum agenesis as well as high T2 and FLIAR signal of the deep white matter with diffusion restriction at DWI in agreement with Manohar et al. [1], Karimzadeh [4], Nicholas et al. [9], and Mohammad et al. [10] who reported similar imaging findings.

The limitation of the study was the small number of patients with possible bias due to the narrow selection criteria, but this can be explained by the fact that these types of brain abnormalities are not frequently seen; a large multicenter study is recommended with additional MRI sequences, e.g., MR spectroscopy for better characterization and possible differentiation of various causes of non-traumatic, non-infective brain encephalopathy among NICU/PICU patients.

\section{Conclusion}

Much attention should be paid to pediatric nontraumatic brain injuries. MRI is a safe modality and should be the first radiological investigation if neurological causes are suggested but should be aided by meticulous clinical evaluation and dedicated laboratory investigations for proper characterization and possible differentiation of various causes of non-traumatic, noninfective brain encephalopathy among NICU/PICU patients. When interpreting MRI, it is essential to have thorough relevant clinical data, gestational age at birth which is prognostic of the pattern of hypoxic-ischemic injury, and the time lag between the onset of HII and the time of performing the MR study.

\section{Abbreviations}

HII: Hypoxic ischemia injury; nTBI: Non-traumatic brain injuries;

PVL: Periventricular leukomalacia; BTBGD: Biotin-thiamine-responsive basal ganglia disease; MELAS: Mitochondrial encephalopathy with lactic acidosis and stroke-like episodes syndrome; NKH: Non-ketotic hyperglycinemia; NICU: Neonatal intensive care; PICU: Pediatric intensive care

\section{Acknowledgements \\ No acknowledgements.}

\section{Authors' contributions}

$\mathrm{HT}$ conceived of the study, participated in its design and coordination, drafted the manuscript, and carried out radiological results. MN participated in the design of the study and sequence alignment as well as participated in radiological/surgical correlation and helped in drafting the results. All authors have read and approved the manuscript.

\section{Funding}

No funding sources.

\section{Availability of data and materials}

The datasets used and analyzed during the current study are available from the corresponding author on reasonable request.

\section{Ethics approval and consent to participate}

The study was approved by the ethical committee of the Faculty of Medicine, Zagazig University. The date of the approval of the protocol was December 2017 from the Department of Radiology, Faculty of Medicine, Zagazig University. The committee has no reference number, only the date.

\section{Consent for publication}

The local ethics committee of our institution ruled that the informed consent from the legal guardian was not needed as this is a retrospective study and all patients' medical information were hidden.

\section{Competing interests}

Thw authors declare that they have no competing interests.

Received: 28 December 2019 Accepted: 17 March 2020

Published online: 22 April 2020

\section{References}

1. Shroff MM, Soares-Fernandes JP, Raybaud C (2010) MR imaging for diagnostic evaluation of encephalopathy in the newborn. RadioGraphics 30: 763-780

2. Cheon J-E, Kim I-O, Hwang YS, Kim KJ, Wang K-C, Cho B-K, Chi JG, Kim CJ, Kim WS, Yeon KM (2002) Leukodystrophy in children: a pictorial review of MR imaging features. RadioGraphics 22:461-476

3. Ibrahim M, Parmar HA, Hoefling N, Srinivasan A (2014) Inborn errors of metabolism: combining clinical and radiologic clues to solve the mystery. AJR 203:W315-W327

4. Karimzadeh $P$ (2015). Approach to neurometabolic diseases from a pediatric neurological point of view. Iran J Child Neurol; Winter; 9(1): 1-16.

5. Alabdulqader MA, AI HS (2018) Biotin-thiamine-responsive basal ganglia disease: case report and follow-up of a patient with poor compliance. Child Neurology Open 5:1-4. https://doi.org/10.1177/2329048X18773218

6. Tabarki B, Alfadhel M, AlShahwan S, Hundallah K, AlShafi S, AlHashem A (2015) Treatment of biotin-responsive basal ganglia disease: open comparative study between the combination of biotin plus thiamine versus thiamine alone. Eur J Paediatr Neurol 19(5):547-552

7. Sremba LJ, Chang RC, Elbalalesy NM, Cambray-Forker EJ, Abdenur JE (2014) Whole exome sequencing reveals compound heterozygous mutations in SLC19A3 causing biotin-thiamine responsive basal ganglia disease. Mol Genet Metab Rep 1:368-372

8. Thomas B, Al Dossary N, Widjaja E (2010) MRI of childhood epilepsy due to inborn errors of metabolism. AJR 194:W367-W374

9. Stence NV, Fenton LZ, Levek C, Tong S et al (2018) Brain imaging in classic non ketotic hyperglycinemia: quantitative analysis and relation to phenotype. J Inherit Metab Dis 42:438-450 
10. Mohammad S.A., Abdelkhalek H.S. (2019). Non ketotic hyperglycinemia: spectrum of imaging findings with emphasis on diffusion-weighted imaging. Neuroradiology;59 (Nov), Issue 11:1155-1163

11. Butler CJ, Likeman M, Mallick AA (2017) Distinctive magnetic resonance imaging findings in neonatal non ketotic hyperglycinemia. Pediatr Neurol 72:90-91

12. Zubarioglu T, Kiykim E, Cansever MS et al (2016) Neonatal non ketotic hyperglycinemia: diffusion-weighted magnetic resonance imaging and diagnostic clues. Acta Neurol Belg 116:671-673

13. Fagon JY, Chastre J, Novara A, Medioni P, Gibert C (1993) Characterization of intensive care unit patients using a model based on the presence or absence of organ dysfunctions and/or infection: the ODIN model. Intensive Care Med. 19:137-144

14. Chan V, Pole JD, Keightley M, Mann RE, Colantonio A (2016) Children and youth with non-traumatic brain injury: a population based perspective. BMC Neurology 16:110. https://doi.org/10.1186/s12883-016-0631-2

15. Chen A, Zagorski B, Chan V, Parsons D, Vander Laan R, Colantonio A (2013) Acute care alternate level of care days due to delayed discharge for traumatic and non-traumatic brain injuries. Healthcare Policy 7(4):41-55

16. Ghei SK, Zan E, Nathan JE, Choudhri A, Tekes A, Huisman TAGM, Izbudak I (2014) MR imaging of hypoxic-ischemic injury in term neonates: pearls and pitfalls. RadioGraphics 34:1047-1061

17. Sen K, Agarwal R (2018) More than meets the eye: infant presenting with hypoxic ischaemic encephalopathy. BMJ Case Rep. https://doi.org/10.1136/ bcr-2017-223614

\section{Publisher's Note}

Springer Nature remains neutral with regard to jurisdictional claims in published maps and institutional affiliations.

\section{Submit your manuscript to a SpringerOpen ${ }^{\circ}$ journal and benefit from:}

- Convenient online submission

- Rigorous peer review

- Open access: articles freely available online

High visibility within the field

- Retaining the copyright to your article

Submit your next manuscript at $\boldsymbol{\nabla}$ springeropen.com 